\title{
Integron-Mediated Antibiotic Resistance in Acinetobacter baumannii Isolated from Intensive Care Unit Patients, Babol, North of Iran
}

\author{
Mitra Deylam Salehi, ${ }^{1}$ Elaheh Ferdosi-Shahandashti, ${ }^{2}$ Yosef Yahyapour, ${ }^{3}$ Soraya Khafri, \\ Abazar Pournajaf, ${ }^{5}$ and Ramazan Rajabnia ${ }^{3}$ \\ ${ }^{1}$ Department of Microbiology, Faculty of Medicine, Babol University of Medical Sciences, Babol, Iran \\ ${ }^{2}$ Department of Medical Biotechnology, Faculty of Advanced Technologies in Medicine (SATiM), Tehran University of Medical Sciences, \\ Tehran, Iran \\ ${ }^{3}$ Infectious Diseases and Tropical Medicine Research Center, Babol University of Medical Sciences, Babol, Iran \\ ${ }^{4}$ Department of Biostatistics and Epidemiology, Faculty of Medicine, Babol University of Medical Sciences, Babol, Iran \\ ${ }^{5}$ Department of Microbiology, Faculty of Medicine, Iran University of Medical Sciences, Tehran, Iran
}

Correspondence should be addressed to Ramazan Rajabnia; ramazan69@yahoo.com

Received 6 December 2016; Revised 12 February 2017; Accepted 27 February 2017; Published 19 July 2017

Academic Editor: Paul M. Tulkens

Copyright ( 2017 Mitra Deylam Salehi et al. This is an open access article distributed under the Creative Commons Attribution License, which permits unrestricted use, distribution, and reproduction in any medium, provided the original work is properly cited.

\begin{abstract}
Background. We investigated the integron types and their relation with antibiotic resistance among A. baumannii isolates collected from intensive care unit patients, Babol, north of Iran. Methods. In this cross-sectional study, a total of 73 bronchoalveolar lavage samples were obtained from patients in ICU. Susceptibility testing was performed by disk diffusion method. Types of integrons were identified by an integrase gene PCR. Results. In total, $47.9 \%$ A. baumannii isolates were recovered from the BAL samples. All isolates were resistant to ceftazidime. $91.4 \%$ and $58.3 \%$ of isolates were MDR and XDR, respectively. The rate of colistin resistance with the $E$-test was $5.7 \%$. Molecular analysis of class I, II, and III integrons showed that $25.7 \%, 88.6 \%$, and $28.6 \%$ of the isolates carried the intI, intII, and intIII genes, respectively. Discussion. Our results show that different classes of integrons are commonly spread among A. baumannii strains and these genomic segments can play an important role in the acquisition of MDR and XDR phenotypes. So monitoring drug resistance in A. baumannii isolates with the use of int gene PCR is very important to plan specific infection control measures to prevent the spread of MDR-AB and XDR-AB in Iran's hospitals.
\end{abstract}

\section{Introduction}

Multidrug-resistant Acinetobacter baumannii (MDR-AB) is an important opportunistic pathogen responsible for severe hospital-acquired infections (HAIs), particularly patients admitted to the intensive care unit (ICU) [1]. MDR-AB infections especially pneumonia and bacteremia show a high mortality rate (30\% to $75 \%)$ and require prolonged hospital stays in intensive care units (ICUs) [2]. In the ICU, the mortality rate associated with $A$. baumannii is $54 \%$. The majority of infections are originated from epidemic outbreaks. Appearance of extensively drug-resistant $A$. baumannii (XDR-AB) limits the therapeutic options and causes a serious concern to HAI control. For a period of 5 years (2005-2010), the frequency of XDR-AB in clinical isolates was increased from $15 \%$ to more than $41 \%$. Control of MDR-AB and XDR-AB infections is an important issue for clinical microbiologists and physicians. Treatment of $A$. baumannii has become difficult because many isolates are now resistant to a wide range of antimicrobial agents $[3,4]$.

An important factor that influences the development of multiresistance phenotype is the acquisition of mobile genetic elements (MGEs). Antibiotic-resistant elements in Acinetobacter strains are commonly carried on MGEs, such as the $\mathrm{R}$ plasmids, transposons (TEs), integrons (Int), and genomic islands (GEIs) [5]. Integrons are conserved sequences $\left(3^{\prime}\right.$-CS 
and $\left.5^{\prime}-\mathrm{CS}\right)$ of DNA that are able to acquire gene cassettes, which can carry antibiotic resistance genes, by site-specific recombination [6]. These genetic segments are considered by the presence of an intI gene (integrase), a recombination site (attI), and a promoter $\left(\mathrm{P}_{\mathrm{C}}\right)$ [7]. The most common types of integrons are the transportable class I (Tn402 derivatives) integron, followed by class II and class III integrons, respectively. Class I integrons harbor numerous antimicrobial resistance gene cassettes encoding broad-spectrum $\beta$ lactamase, $d f r$ (dihydroflavonol-4-reductase/trimethoprim), qacE $\Delta 1$ (disinfectants and tetravalent ammonium compounds), sul1 (sulfonamide), and aminoglycoside-modifying enzymes (AMEs). Integrons of class II are located in Tn7 and $3^{\prime}$-CS which contains five $t n s$ genes and are responsible for the mobility of TE. Integrons of class III located in transposons have also been described, but the $3^{\prime}$-CS is still not well defined [8].

MDR phenotype occurs in A. baumannii when different integrin-borne antibiotic resistance elements coexist, giving rise to MDR gene cassettes $[9,10]$. Due to the high prevalence of this infection, as well as various profiles of drug resistance in different geographical areas, a study on the prevalence and antibiotic resistance pattern in different part of the world is essential. These data would provide useful information on the spread of resistance elements and the possibility of choosing appropriate treatment strategies. So, the aim of this study was to determine the frequency of type I, II, and III integrons in A. baumannii isolates recovered from bronchoalveolar lavage (BAL) samples obtained from hospitalized patients in ICU ward, Babol, north of Iran [11].

\section{Materials and Methods}

2.1. Setting. The Ayatollah Rouhani hospital is a 372-bed hospital and is one of the most equipped teaching therapeutic centers affiliated to the Babol Medical University in north of Iran. This study was approved by the ethical committee of Babol University of Medical Sciences for Research Analysis. In this analytical cross-sectional study, based on previous studies and confidence interval $95 \%$ and using equation $n=$ $z^{2} P(1-P) / d^{2}$, a total of 73 nonrepetitive and nonduplicative BAL samples were collected from ICU patients from May to November 2015.

2.2. Inclusion Criteria. Clinical findings of pneumonia are as follows: new radiographic infiltrate and two of the three subsequent criteria: fever [body temperature $\geq 38.0^{\circ} \mathrm{C}$ ] or hypothermia temperature $<35.0^{\circ} \mathrm{C}$ [rqb], purulent bronchial secretion, and WBCs $>10,000 / \mathrm{mm}^{3}$ or $<3,000 / \mathrm{mm}^{3}[12]$.

2.3. Exclusion Criteria. Contraindications to bronchoscopy include the following: (1) Po $<75 \mathrm{~mm} \mathrm{Hg}$ even with an inspired oxygen fraction of $100 \%$; (2) acute cardiac arrhythmia; and (3) bronchospasm [12].

2.4. Chest Radiograph (Chest X-Ray). A portable chest radiograph was obtained prior to, and on the same day as, bronchoscopic sampling. Infiltration of lung (chest X-ray) was included as a criterion for hospital admission. The radiograph was reviewed and interpreted by a staff radiologist and pulmonary disease specialist [12].

2.5. Procedure and Specimen Collection (BAL Collection). Patients were commonly sedated with IV midazolam before bronchoscopic procedure. The fraction of inspired oxygen was increased to $100 \%$, and positive end-expiratory pressure was limited to $8 \mathrm{~cm} \mathrm{H}_{2} \mathrm{O}$. The bronchoscope used was the Pentax FB15X (Pentax Instruments, Tokyo, Japan). Bronchoscopic BAL samples were collected by pulmonologist by wedging the tip of a fiber optic bronchoscope in the subsegmental bronchus of the most compromised lobe seen in chest $\mathrm{X}$-ray or, in cases of diffuse radiologic presentation, in the posterior bronchus of the lower lobe. As little topical lidocaine as possible was used so as not to interfere with bacterial growth (never $>20 \mathrm{mg}$ per bronchus). Aspiration of secretions by the bronchoscope was avoided. This procedure was repeated in the contralateral lung, and samples were preserved at ambient temperatures or $4^{\circ} \mathrm{C}$ before being transported to the microbiology laboratory within $2 \mathrm{~h}$ of collection $[12,13]$.

2.6. Quantitative Cultures. Quantitative cultures were processed according to the standard laboratory protocol. Sample pairs collected were diluted in tryptic soy broth (Merck, Co., Germany) plated at final dilutions of $10^{-3}$ and $10^{-4}$ onto eosin methylene blue agar, chocolate agar and blood agar (Merck, Co., Germany) plates and then incubated at $35^{\circ} \mathrm{C}$ under $5 \%$ $\mathrm{CO}_{2}$. Gram staining was performed on 1 drop of undiluted BAL fluid. Final colony counts were determined after $48 \mathrm{~h}$. Potential pathogens present at $\geq 1 \times 10^{4} \mathrm{CFU} / \mathrm{ml}$ were considered clinically significant, and subsequent identification and antimicrobial susceptibility testing were performed. Isolates detected at $\geq 1 \times 10^{3}$ and $<1 \times 10^{4} \mathrm{CFU} / \mathrm{ml}$ were presumptively identified, and antimicrobial susceptibility testing was not performed. The following were considered etiologic agents of pneumonia: (1) bacteria with $>10^{4} \mathrm{CFU} / \mathrm{mL}$ in BAL quantitative culture (according to clinical judgment, $>10^{3} \mathrm{CFU} / \mathrm{mL}$ was considered positive) and (2) demonstration by direct microscopic examination of BAL fluid $[12,13]$.

2.7. Bacterial Isolates. After transferring all samples to the Department of Microbiology in the medicine faculty, $A$. baumannii strains were recognized based on conventional biochemical and microbiological tests including Gram staining, oxidase and catalase test, motility, oxidation of glucose, hydrolysis of esculin, decarboxylation of lysine, hydrolysis of arginine, reduction of nitrate, citrate utilization, oxidative/fermentative glucose $(\mathrm{O} / \mathrm{F})$ test, and growth ability at $44^{\circ} \mathrm{C}$. Negative result for oxidase test, no motility, nonfermentation, and growth in temperature of $42-44^{\circ} \mathrm{C}$ were considered as the elementary criteria for A. baumannii recognition [14]. In order to confirm the identity of strains, the presence of gyrB gene was assessed using PCR $[15,16]$. The isolates were preserved in $-80^{\circ} \mathrm{C}$ in brain-heart infusion broth (Merck, Co., Germany) containing glycerol 50\% v/v until the molecular analysis. 
TABLE 1: Primer sequences, PCR product sizes, and PCR programs for replication of class I, II, and III integrons.

\begin{tabular}{|c|c|c|c|c|}
\hline Genes & Primer sequence $\left(5^{\prime} \rightarrow 3^{\prime}\right)$ & PCR product (bp) & PCR program & Reference \\
\hline IntI-I & $\begin{array}{l}\text { F-TCTCGGGTAACATCAAGG } \\
\text { R-AGGAGATCCGAAGACCTC }\end{array}$ & 243 & $\begin{array}{c}5 \mathrm{~min} \text { at } 94^{\circ} \mathrm{C} ; 35 \mathrm{cycles}\left(1 \mathrm{~min} \text { at } 94^{\circ} \mathrm{C} \text {, }\right. \\
\left.1 \mathrm{~min} \text { at } 53^{\circ} \mathrm{C} \text {, and } 30 \mathrm{sec} \text { at } 72^{\circ} \mathrm{C}\right) ; 5 \mathrm{~min} \\
\text { at } 72\end{array}$ & [9] \\
\hline IntI-II & $\begin{array}{l}\text { F-TTATTGCTGGGATTAGGC } \\
\text { R-ACGGCTACCCTCTGTTATC }\end{array}$ & 233 & $\begin{array}{c}5 \mathrm{~min} \text { at } 94^{\circ} \mathrm{C} ; 30 \text { cycles }\left(1 \mathrm{~min} \text { at } 94^{\circ} \mathrm{C} \text {, }\right. \\
\left.1 \mathrm{~min} \text { at } 50^{\circ} \mathrm{C} \text {, and } 1 \mathrm{~min} \text { at } 72^{\circ} \mathrm{C}\right) ; 10 \mathrm{~min} \\
\text { at } 72^{\circ}\end{array}$ & [10] \\
\hline IntI-III & $\begin{array}{l}\text { F-AGTGGGTGGCGAATGAGTG } \\
\text { R-TGTTCTTGTATCGGCAGGTG }\end{array}$ & 600 & $\begin{array}{c}5 \mathrm{~min} \text { at } 94^{\circ} \mathrm{C} ; 30 \text { cycles }\left(1 \mathrm{~min} \text { at } 94^{\circ} \mathrm{C} \text {, }\right. \\
\left.1 \mathrm{~min} \text { at } 50^{\circ} \mathrm{C} \text {, and } 1 \mathrm{~min} \text { at } 72^{\circ} \mathrm{C}\right) ; 10 \mathrm{~min} \\
\text { at } 72^{\circ} \mathrm{C}\end{array}$ & [10] \\
\hline
\end{tabular}

2.8. Antimicrobial Susceptibility Testing. Antimicrobial susceptibility testing was performed on Mueller-Hinton agar (MHA) (Merck, Co., Germany) by agar disk diffusion (DD) method as recommended by the Clinical and Laboratory Standards Institute (CLSI document M100-S14) [17]. The tested antibiotics were as follows: amikacin (AK; $30 \mu \mathrm{g})$, ciprofloxacin (CP; $5 \mu \mathrm{g}$ ), ceftazidime (CAZ; $30 \mu \mathrm{g})$, gentamicin (GM; $10 \mu \mathrm{g})$, imipenem (IMP; $10 \mu \mathrm{g})$, meropenem (MER; $10 \mu \mathrm{g}$ ) and piperacillin/tazobactam (PTZ; 100/10 $\mu \mathrm{g}$ ) (MAST diagnostics, Merseyside, UK). A. baumannii ATCC 17978 was used as a positive quality control (PQC) and $P$. aeruginosa ATCC 25853 and E. coli ATCC 25922 were used as a negative quality control (NQC) in this study.

2.9. Minimum Inhibitory Concentration (MIC). All isolates were tested for colistin (CS) susceptibility by E-test according to the manufacturer's guidelines (Liofilchem SRL, Italy). Suspension of each isolate in Mueller-Hinton broth, adjusted to the density of a $0.5 \mathrm{McF}$ arland standard, was swabbed in three directions to ensure uniform growth onto MuellerHinton agar plates. An E-test CS strip (ranging from 0.016 to $256 \mathrm{mg} / \mathrm{ml}$ ) with interpretative criteria (susceptible (S), $\leq 2 \mu \mathrm{g} / \mathrm{ml}$; resistant $(\mathrm{R}), \geq 4 \mu \mathrm{g} / \mathrm{ml}$ ) was used for each plate when the agar surface was completely dry, and the plates were incubated at $35^{\circ} \mathrm{C}$ for $16-20 \mathrm{~h}$. The minimum inhibitory concentration (MIC) value was considered at the point of complete inhibition of all growth, including hazes. The interpretive criteria used were those established in Clinical and Laboratory Standards Institute standard [14].

2.10. PCR Amplification and Sequencing. The PCR technique were performed by the DNA amplification device master cycler gradient (Eppendorf Co., Hamburg, Germany) for detection of intI-I, intI-II, and intI-III genes. Whole-cell (genomic) DNA was extracted from each strain using a high pure PCR template preparation kit (Roche Co. in Germany). The integron encoding gene classes and PCR programs are listed in Table 1. PCR was performed in a whole volume of $25 \mu \mathrm{l}$. PCR mixture contained $2 \mu \mathrm{l}$ of 10x PCR buffer, 1.5 DNA template, $0.8 \mu \mathrm{l} \mathrm{MgCl}_{2}, 0.6 \mu \mathrm{l} \mathrm{dNTPs}, 1.5 \mu \mathrm{l}$ of each primer, 0.7 IU of Taq DNA polymerase (Ampliqon Co., Denmark), and $14.9 \mu \mathrm{l}$ of sterile distilled water. Amplification products were electrophoresed on $1.5 \%$ agarose gels at $5 \mathrm{~V} / \mathrm{cm}$. Gels were stained with ethidium bromide $(\mathrm{EtBr})(0.5 \mu \mathrm{g} / \mathrm{ml})$ and visualized on a UV trans-illuminator (Vilber Lourmat,
Cedex, France). The amplicon sizes were determined by comparison with a DNA size marker (100-bp DNA ladder, Fermentas). Direct sequencing of the PCR products was performed in both directions using an ABI3730XL DNA analyzer (Applied Biosystems, Forster, USA). Nucleotide sequence data were analyzed at the National Center for Biotechnology Information (NCBI), available at the website (http://blast.ncbi.nlm.nih.gov/Blast.cgi).

2.11. Statistical Analysis. SPSS version 23 (SPSS, Inc., Chicago, IL, USA) was employed for statistical analysis. Descriptive statistics and Pearson's chi-square tests were used to evaluate the correlation between various classes of integrons and antimicrobial resistance. Statistical significance was defined as $P$ value less than 0.05 .

\section{Results}

Mean of patients ages was 54.5 years (range, 17 to 92 years). $32(43.8 \%)$ of them were female and $41(56.2 \%)$ were male. The mean APACHE II (acute physiology and chronic health evaluation II) index was 16.2 at ICU admission (range, 7 to 31). Mean duration of hospitalization until BAL performance was 14.3 days (range, 4 to 48 days). The main reasons for admission to ICU were as follows: neurologic emergencies in 23 patients (31.5\%); respiratory failure in $19(26.0 \%)$; cardiocirculatory emergencies in $14(19.2 \%)$; other infections in $3(4.1 \%)$; hematologic disorders in 3 (4.1\%); trauma in 3 (4.1\%); septic shock in $2(2.7 \%)$; renal failure in $2(2.7 \%)$; diabetes in $2(2.7 \%)$; malignancy in $1(1.4 \%)$, and acute abdomen in 1 (1.4\%).

Forty-nine of 73 BALs (67.1\%) presented $>10^{4} \mathrm{CFU} / \mathrm{mL}$ in BAL quantitative culture. The three BALs (4.8\%) that presented between $10^{3}$ and $10^{4} \mathrm{CFU} / \mathrm{mL}$ were also considered positive. Total positivity was 52 of 73 (71.2\%); 49 of these 52 positive $(67.1 \%)$ had $>10^{4} \mathrm{CFU} / \mathrm{mL}$. There were no cases of bacterial growth with $<10^{3} \mathrm{CFU} / \mathrm{mL}$. Twenty-one cases (28.7\%) did not present bacterial growth (Table 2).

A. baumannii isolates $(n=35 ; 47.9 \%)$ were recovered from the BAL samples $(n=73)$. The highest resistance rate was related to CAZ (100\%) (Table 3 ). In the current study, $\mathrm{MDR}-\mathrm{AB}$ is defined as resistance to more than three classes of antibiotics. XDR-AB is regarded as the isolate that is resistant to 3 classes of antibiotics defined above (MDR) as well 
TABLE 2: Bacterial etiologic agents.

\begin{tabular}{lccc}
\hline \multirow{2}{*}{ Agents } & \multicolumn{3}{c}{ All BALs } \\
& Mono (\%) & Poly (\%) & Total (\%) \\
\hline A. baumannii & $30(41.1 \%)$ & $5(6.8 \%)$ & $35(48 \%)$ \\
Pseudomonas aeruginosa & $3(4.1 \%)$ & $1(1.9 \%)$ & $4(5.5 \%)$ \\
Staphylococcus aureus & $2(2.7 \%)$ & $2(2.7 \%)$ & $4(5.5 \%)$ \\
Klebsiella pneumoniae & $1(1.4 \%)$ & $0(0.0 \%)$ & $1(1.4 \%)$ \\
Escherichia coli & $0(0.0 \%)$ & $1(1.4 \%)$ & $1(1.4 \%)$ \\
Enterobacter cloacae & $0(0.0 \%)$ & $1(1.4 \%)$ & $1(1.4 \%)$ \\
Serratia marcescens & $0(0.0 \%)$ & $2(2.7 \%)$ & $2(2.7 \%)$ \\
Stenotrophomonas maltophilia & $1(1.4 \%)$ & $0(0.0 \%)$ & $1(1.4 \%)$ \\
MRCoNS & $1(1.4 \%)$ & $0(0.0 \%)$ & $1(1.4 \%)$ \\
Streptococcus pneumoniae & $2(2.7 \%)$ & $0(0.0 \%)$ & $2(2.7 \%)$ \\
\hline
\end{tabular}

Mono $=$ unique isolated agent; Poly $=$ polymicrobial infection; MRCoNS $=$ methicillin resistant coagulase-negative Staphylococcus.

TABle 3: Antibiotics resistance patterns among Acinetobacter isolates.

\begin{tabular}{|c|c|c|c|}
\hline Antibiotics & $\mathrm{R}(\%)$ & I (\%) & S (\%) \\
\hline Amikacin (AN) & $32(91.4)$ & $0(0.0)$ & $3(8.6)$ \\
\hline Gentamicin (GM) & $30(85.7)$ & $1(2.9)$ & $4(11.4)$ \\
\hline Ciprofloxacin (CP) & $33(94.3)$ & $0(0.0)$ & $2(5.7)$ \\
\hline Ceftazidime (CAZ) & $35(100)$ & $0(0.0)$ & $0(0.0)$ \\
\hline Imipenem (IPM) & $33(94.3)$ & $2(5.7)$ & $0(0.0)$ \\
\hline Meropenem (MEN) & $33(94.3)$ & $1(2.9)$ & $1(2.9)$ \\
\hline $\begin{array}{l}\text { Piperacillin- } \\
\text { tazobactam } \\
\text { (PIP-TZ) }\end{array}$ & $32(91.4)$ & $1(2.9)$ & $2(5.7)$ \\
\hline
\end{tabular}

$\mathrm{I}=$ intermediate; $\mathrm{R}$ = resistance; $\mathrm{S}=$ susceptible.

as to carbapenems. Finally, pan-drug-resistant A. baumannii (PDR-AB) is the $\mathrm{XDR}$ isolate that is also resistant to tigecycline and polymyxins. According to the results of the antimicrobial susceptibility test, $91.4 \%$ and $58.3 \%$ of the isolates were MDR and XDR. The rates of CS resistance with the $E$-test method in all A. baumannii studied strains were determined $5.7 \%$ ( $n ; 2$ of 35$)$ (Figure 1$)$.

Molecular detection of class I, II, and III integrons was performed by amplification of intI, intII, and intIII genes in all A. baumannii strains using PCR method. The results revealed that $94.3 \%(n ; 33 / 35)$ of the isolates carried the int gene. Molecular analysis of class I, II, and III integrons showed that $25.7 \%(n ; 9), 88.6 \%(n ; 31)$, and $28.6 \%(n ; 10)$ of isolates carried the intI, intII, and intIII genes, respectively. The coexistence of intI/intII and intI/intII/intIII in isolates was in order $22.9 \%(n ; 8)$ and $8.6 \%(n ; 3)$. Two $(5.7 \%)$ of all isolates were int-negative. The correlation of class I, II, and III integrons and antibiotic resistance profile are shown in Table 4. Nucleotide sequence data reported in the current study has been deposited in the PubMed/NCBI/GenBank nucleotide sequence databank under the accession numbers KX122025.1, KX122026.1, KX122027.1, KX122028.1, and KX122029.1.

\section{Discussion}

A. baumannii is an important opportunistic bacterium causing severe HAIs with high mortality rates due to its wide drug resistance. Integrons are vital in enabling the A. baumannii genome to capture and accumulate many antimicrobial resistance genes. Because of the MDR phenotype and the trend to spread in the hospital environment, A. baumannii has a special clinical significance requiring epidemiological monitoring as a measure to control the spread of HAIs [18].

In line with Gomes et al. [12], Zaccard et al. [13], and Nomanpour et al. [19] studies, considering $10^{4} \mathrm{CFU} / \mathrm{mL}$ as the cutoff and including the cases with bacterial growth between $10^{3}$ and $10^{4} \mathrm{CFU} / \mathrm{mL}, 28$ of all 52 positive BALs $(53.8 \%)$ presented more than one isolate, which means polymicrobial infections. The most frequently encountered pathogens were associated with two different strains of A. baumannii (48\%) and between $P$. aeruginosa (5.5\%) and MRSA (5.5\%), with three cases of each association.

Our findings showed that all these strains were resistant to CAZ. DD method results showed $91.4 \%$ and $58.3 \%$ of the isolates were MDR and XDR, respectively. These results are in agreement with the Ghajavand et al. study [20]. In a parallel study piloted in Taipei, Taiwan, 25 XDR-AB were found during two years of study [21]. This contrast may be due to the geographical distance and/or the different levels of hygiene. The majority of the strains were sensitive to CS, regarded as the last resort against $M D R-A B$, probably due to the fact that CS has not been broadly used in Iran; thus, the corresponding selection pressure has not been established on this bacterial species. E-test MIC value showed that $5.7 \%$ $(n ; 2)$ of isolates were resistant to CS. This data are similar to Vakili et al. [14]. All CS-resistant isolates were positive for class II integron. No significant association was observed between CS-resistant isolates and class II integron.

Integrase-encoding genes were found in 33 (94.3\%) of the 35 isolates. Our data showed that the prevalence of class I, II, and III integrons was $25.7 \%, 88.6 \%$, and $28.6 \%$, respectively. In the strains that had all the three classes of integrons, resistance to all the antibiotics tested was observed. Two samples (5.71\%) did not contain any integrons but were still resistant to $\mathrm{CP}$ and $\mathrm{CAZ}$, which indicates that resistance genes could also be transmitted by other elements such as plasmids, TEs, and bacteriophages. In the study carried by Ramírez et al., the frequency of class I and II integrons was $42 \%$ and $68 \%$, respectively [22]. Also, Kamalbeik et al. showed that $7.5 \%$ and $67.5 \%$ of the strains contained class I and II integrons, respectively [23]. In the studies carried out by Ramírez et al. [24] and Martins et al. [25], the prevalence of class II Integron was $23 \%$ and $41.7 \%$, respectively. In the research conducted by Taherikalani et al. (Tehran at 2012) [26] and Moammadi et al. (west of Iran at 2012) [27] the frequency of class I, II, and III integrons was $85 \%, 14 \%$, and $0 \%$, and $97 \%, 31 \%$, and $0 \%$, respectively. These findings are in contrast with our results and may be related to the difference in source of samples, level of hygiene, and geographical areas. So, it might be due to stiff search-and-destroy and surveillance policies, as well as control in antibiotic prescription. 


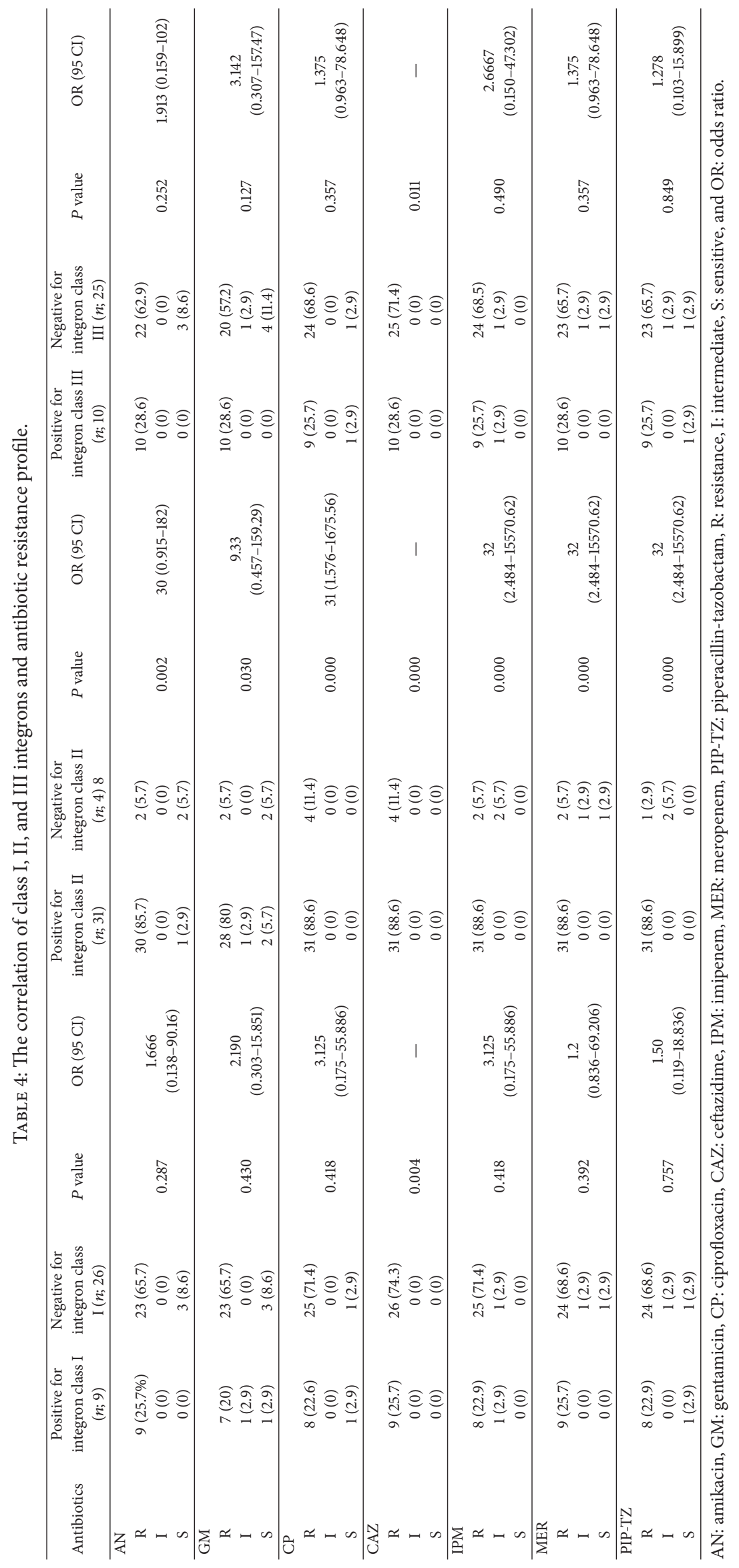




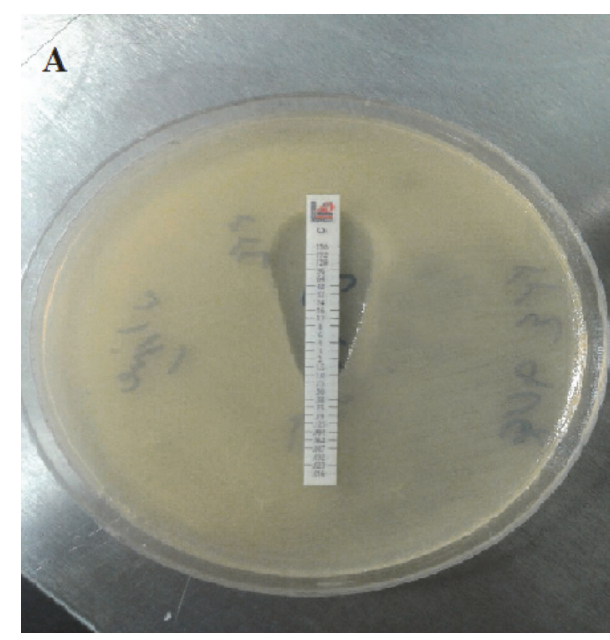

(a)

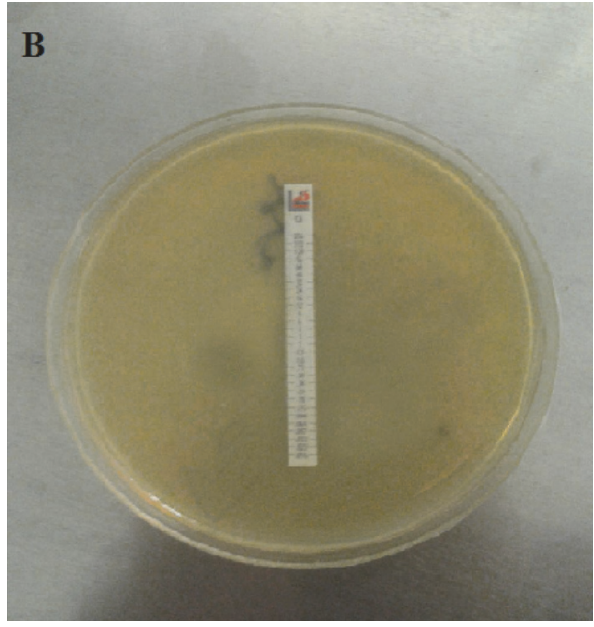

(b)

FIGURE 1: E-test MIC value of colistin. (a) Colistin-susceptible A. baumannii (interpretative criteria; susceptible (S), $\leq 2 \mu \mathrm{g} / \mathrm{ml}$ ). (b) Colistinresistant A. baumannii (interpretative criteria; resistant (R), $\geq 4 \mu \mathrm{g} / \mathrm{ml}$ ). Colistin sulfate (CS), ranging from 0.016 to $256 \mathrm{mg} / \mathrm{ml} \mathrm{produced}$ by Liofilchem SRL, Roseto degli Abruzzi, Italy.

Integron-positive A. baumannii isolates showed higher antibiotic resistance rate compared to integron-negative strains. All the isolates with class II integron had a significant relationship with resistance to all the tested antibiotics which could be due to the gene cassettes that encoded resistance to these antibiotics. According to our results, there was a significant correlation between the presence of class I, II, and III integrons and resistance to CAZ. Lin et al. [28] and Mirnejad et al. [29] observed a significant correlation between the presence of integrons and resistance to $\mathrm{CP}$, ofloxacin, cefepime, CAZ, aztreonam, AN, and norfloxacin. Koeleman et al. indicated a significant relationship between the presence of integrons and resistance to $\mathrm{AN}, \mathrm{CP}$, and CAZ [30]. The results of Gaur et al. confirmed the results of our study and showed correlation between the presence of integrons and resistance to AN, cefepime, and CP [31]. In cases where significant relationships between the integrons and antimicrobials resistance were not detected, resistance could be attained by resistance elements that reside on MGEs, such as plasmids, TEs, and phages, indicating substantial HGT from other bacteria [32].

\section{Conclusion}

In general, our results showed that different classes of integrons are commonly spread among A. baumannii strains and these genomic segments can play an important role in the acquisition of MDR and XDR A. baumannii. In this study, regardless of the presence or lack of resistance genes, strong association was observed between the presence of class II integrons and a decreased sensitivity to the many classes of antibiotics which could be challenging, since these structures can move among strains and consequently become resistant to new antimicrobials. So, monitoring drug resistance in A. baumannii isolates with the use of int gene PCR is very important to plan specific infection control measures to prevent the spread of MDR-AB and XDR-AB in Iran's hospitals.

\section{Conflicts of Interest}

No conflicts of interest were reported by the authors of this paper.

\section{Authors' Contributions}

Dr. Ramazan Rajabnia (supervisor) conceived and designed the experiments. Mitra Deylam Salehi performed the experiments. Elaheh Ferdosi-Shahandashti and Dr. Yosef Yahyapour analyzed the data. Statistical analysis and sample size calculation were conducted by Dr. Soraya Khafri and Abazar Pournajaf wrote the paper.

\section{Acknowledgments}

This work was supported by Infection Diseases Research Center (IDRC), Babol University of Medical Sciences (BUMS), Babol, Iran (Contract no. 9339621). The authors would like to thank the Ayatollah Rouhani hospital (Babol, Iran) for providing samples and for their collaboration in this research.

\section{References}

[1] P. K. Sahu, P. S. Iyer, S. H. Barage, K. D. Sonawane, and B. A. Chopade, "Characterization of the algC gene expression pattern in the multidrug resistant Acinetobacter baumannii AIIMS 7 and correlation with biofilm development on abiotic surface," Scientific World Journal, vol. 2014, Article ID 593546, 2014.

[2] D. E. Karageorgopoulos and M. E. Falagas, "Current control and treatment of multidrug-resistant Acinetobacter baumannii infections," The Lancet Infectious Diseases, vol. 8, no. 12, pp. 751$762,2008$. 
[3] V. Manchanda, S. Sanchaita, and N. P. Singh, "Multidrug resistant acinetobacter," Journal of Global Infectious Diseases, vol. 2, no. 3, pp. 291-304, 2010.

[4] R. A. Bonomo and D. Szabo, "Mechanisms of multidrug resistance in Acinetobacter species and Pseudomonas aeruginosa," Clinical Infectious Diseases, vol. 43, supplement 2, pp. S49-S56, 2006.

[5] A. Z. Bialvaei, H. S. Kafil, M. Asgharzadeh, M. Aghazadeh, and M. Yousefi, "CTX-M extended-spectrum $\beta$-lactamaseproducing Klebsiella spp, Salmonella spp, Shigella spp and Escherichia coli isolates in Iranian hospitals," Brazilian Journal of Microbiology, vol. 47, no. 3, pp. 706-711, 2016.

[6] M. Taherikalani, A. Maleki, N. Sadeghifard et al., "Dissemination of class 1, 2 and 3 integrons among different multidrug resistant isolates of Acinetobacter baumannii in Tehran hospitals, Iran," Polish Journal of Microbiology, vol. 60, no. 2, pp. 169174, 2011.

[7] Y. Zhu, Y. Yi, F. Liu et al., "Distribution and molecular profiling of class 1 integrons in MDR Acinetobacter baumannii isolates and whole genome-based analysis of antibiotic resistance mechanisms in a representative strain," Microbiological Research, vol. 169, no. 11, pp. 811-816, 2014.

[8] F. Moradian Kouchaksaraei, E. Ferdosi Shahandashti, Z. Molana et al., "Molecular detection of integron genes and pattern of antibiotic resistance in pseudomonas aeruginosa strains isolated from intensive care unit, Shahid Beheshti hospital, North of Iran," International Journal of Molecular and Cellular Medicine, vol. 1, no. 4, pp. 209-217, 2012.

[9] F. Ahangarkani, R. Rajabnia, E. Shahandashti, M. Bagheri, and M. Ramez, "Frequency of class 1 integron in Escherichia coli strains isolated from patients with urinary tract infections in North of Iran," Mater Sociomed, vol. 27, no. 1, pp. 10-12, 2015.

[10] C. Goldstein, M. D. Lee, S. Sanchez et al., "Incidence of class 1 and 2 integrases in clinical and commensal bacteria from livestock, companion animals, and exotics," Antimicrobial Agents and Chemotherapy, vol. 45, no. 3, pp. 723-726, 2001.

[11] M. Deylam Salehi, E. Ferdosi-Shahandashti, Y. Yahyapour, S. Khafri, A. Pournajaf, and R. Rajabnia, Molecular study ofintegrons class I, II, III and its relation to antibiotic resistance inAcintobacter strains isolated from patients in intensive care hospital Rohani Babol. [A thesis for MSc dissertation], Babol University of medical sciences, Babol, Iran, 2016.

[12] J. C. P. Gomes, W. L. Pedreira, E. M. P. A. Araújo et al., "Impact of BAL in the management of pneumonia with treatment failure: positivity of BAL culture under antibiotic therapy," Clinical Investigations in Critical Care, vol. 118, no. 6, pp. 1739-1746, 2000.

[13] C. R. Zaccard, R. F. Schell, and C. A. Spiegel, "Efficacy of bilateral bronchoalveolar lavage for diagnosis of ventilator-associated pneumonia," Journal of Clinical Microbiology, vol. 47, no. 9, pp. 2918-2924, 2009.

[14] B. Vakili, H. Fazeli, P. Shoaei et al., "Detection of colistin sensitivity in clinical isolates of Acinetobacter baumannii in Iran," Journal of Research in Medical Sciences, vol. 19, pp. S67S70, 2014.

[15] P. G. Higgins, H. Wisplinghoff, O. Krut, and H. Seifert, "A PCRbased method to differentiate between Acinetobacter baumannii and Acinetobacter genomic species 13TU," Clinical Microbiology and Infection, vol. 13, no. 12, pp. 1199-1201, 2007.

[16] W. Kamolvit, P. G. Higgins, D. L. Paterson, and H. Seifert, "Multiplex PCR to detect the genes encoding naturally occurring oxacillinases in Acinetobacter spp," Journal of Antimicrobial
Chemotherapy, vol. 69, no. 4, Article ID dkt480, pp. 959-963, 2014.

[17] "Performance standards for antimicrobial susceptibility testing; twenty-fifth informational supplement," Clinical and Laboratory Standards Institute, vol. 35, no. 3, pp. 44-50, 2015.

[18] F. Perez, A. M. Hujer, K. M. Hujer, B. K. Decker, P. N. Rather, and R. A. Bonomo, "Global challenge of multidrug-resistant Acinetobacter baumanni," Antimicrobial Agents and Chemotherapy, vol. 51, no. 10, pp. 3471-3484, 2007.

[19] B. Nomanpour, A. Ghodousi, A. Babaei, H. Abtahi, M. Tabrizi, and M. M. Feizabadi, "Rapid, cost-effective, sensitive and quantitative detection of Acinetobacter baumannii from pneumonia patients," Iranian Journal of Microbiology, vol. 3, no. 4, pp. 162 $169,2011$.

[20] H. Ghajavand, B. N. Esfahani, S. A. Havaei, S. Moghim, and H. Fazeli, "Molecular identification of Acinetobacterbaumannii isolated from intensive care units and their antimicrobial resistance patterns," Advanced Biomedical Research, vol. 4, no. $110,2015$.

[21] M.-C. Chan, S.-K. Chiu, P.-R. Hsueh, N.-C. Wang, C.-C. Wang, and C.-T. Fang, "Risk factors for healthcare-associated extensively drug-resistant Acinetobacter baumannii infections: a case-control study," PLoS ONE, vol. 9, no. 1, article e85973, 2014.

[22] M. S. Ramírez, M. S. Stietz, E. Vilacoba et al., "Increasing frequency of class 1 and 2 integrons in multidrug-resistant clones of Acinetobacter baumannii reveals the need for continuous molecular surveillance," International Journal of Antimicrobial Agents, vol. 37, no. 2, pp. 175-177, 2011.

[23] S. Kamalbeik, H. Talaie, A. Mahdavinejad, A. Karimi, and A. Salimi, "Multidrug-resistant Acinetobacter baumannii infection in intensive care unit patients in a hospital with building construction: is there an association?" Korean Journal of Anesthesiology, vol. 66, no. 4, pp. 295-299, 2014.

[24] M. S. Ramírez, A. Morales, E. Vilacoba, C. Márquez, and D. Centrón, "Class 2 integrons dissemination among multidrug resistance (MDR) clones of Acinetobacter baumannii," Current Microbiology, vol. 64, no. 3, pp. 290-293, 2012.

[25] N. Martins, R. C. Picão, S. Adams-Sapper, L. W. Riley, and B. M. Moreira, "Association of class 1 and 2 integrons with multidrugresistant Acinetobacter baumannii international clones and Acinetobacter nosocomialis isolates," Antimicrobial Agents and Chemotherapy, vol. 59, no. 1, pp. 698-701, 2015.

[26] M. Taherikalani, A. Maleki, N. Sadeghifars, D. Mohammadzadeh, P. Asadollahi, and M. Emaneini, "Dissemination of class 1,2 and 3 integrons among different multidrug resistant isolates of Acinetobacter baumannii in Tehran hospitals, Iran," Polish Journal of Microbiology, vol. 60, no. 2, pp. 169-174, 2011.

[27] F. Moammadi, M. Arabestani, M. Safari, G. Roshanaii, and M. Alikhani, "Prevalence of class 1, 2 and 3 integrons among extensive drug resistance Acinetobacter baumanii strains isolated from intensive care units in Hamadan, west province," Iranian Journal of Medical Microbiology, vol. 8, no. 3, pp. 8-14, 2014.

[28] M. F. Lin, K. C. Chang, C. Y. Yang et al., "Role of integrons in antimicrobial susceptibility patterns of Acinetobacter baumannii," Japanese Journal of Infectious Diseases, vol. 63, pp. 440-443, 2010.

[29] R. Mirnejad, S. Mostofi, and F. Masjedian, "Antibiotic resistance and carriage class 1 and 2 integrons in clinical isolates of Acinetobacter baumannii from Tehran, Iran," Asian Pacific Journal of Tropical Biomedicine, vol. 3, no. 2, pp. 140-145, 2013. 
[30] J. G. M. Koeleman, J. Stoof, M. W. Van der Bijl, C. M. J. E. Vandenbroucke-Grauls, and P. H. M. Savelkoul, "Identification of epidemic strains of Acinetobacter baumannii by integrase gene PCR," Journal of Clinical Microbiology, vol. 39, no. 1, pp. $8-13,2001$.

[31] A. Gaur, P. Prakash, S. Anupurba, and T. M. Mohapatra, "Possible role of integrase gene polymerase chain reaction as an epidemiological marker: study of multidrug-resistant Acinetobacter baumannii isolated from nosocomial infections," International Journal of Antimicrobial Agents, vol. 29, no. 4, pp. 446-450, 2007.

[32] L. L. Maragakis and T. M. Perl, "Acinetobacter baumannii: epidemiology, antimicrobial resistance, and treatment options," Clinical Infectious Diseases, vol. 46, no. 8, pp. 1254-1263, 2008. 

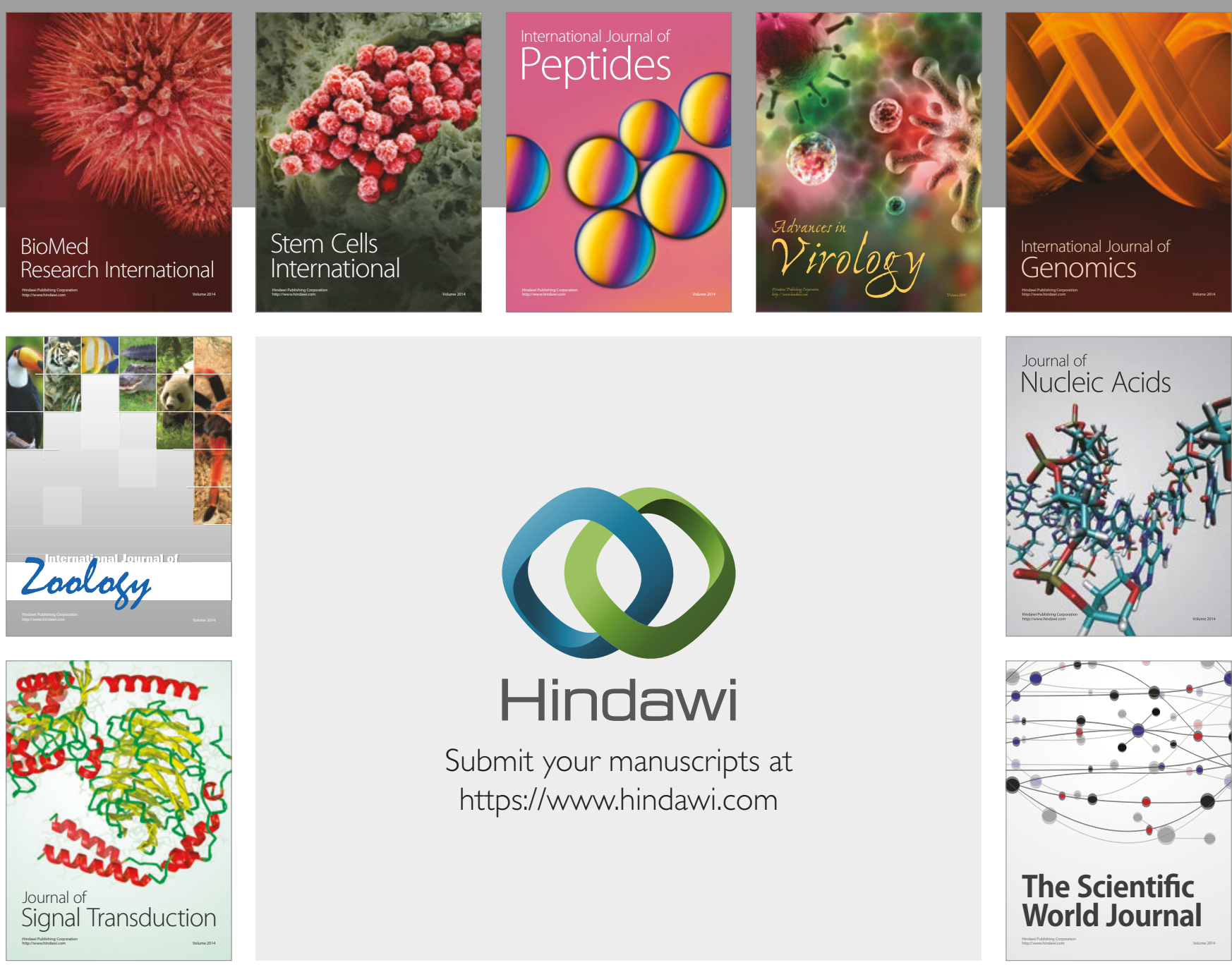

Submit your manuscripts at

https://www.hindawi.com
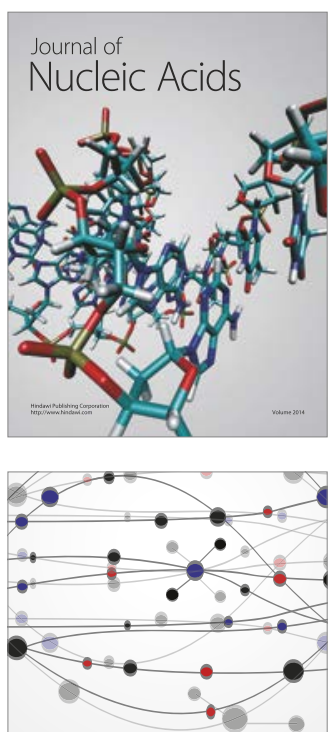

The Scientific World Journal

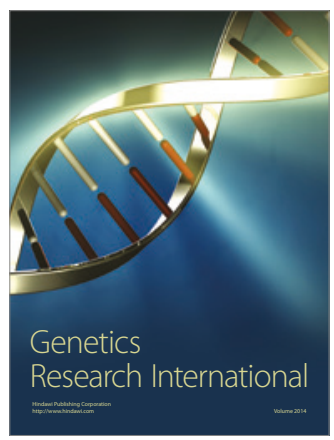

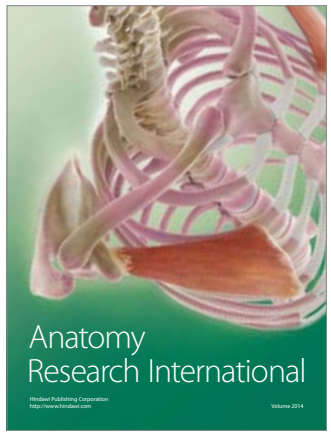

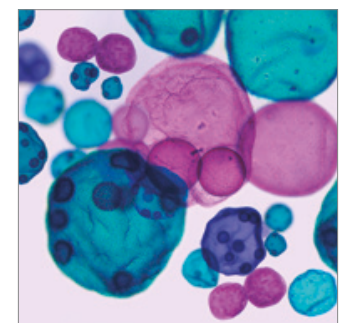

International Journal of Microbiology
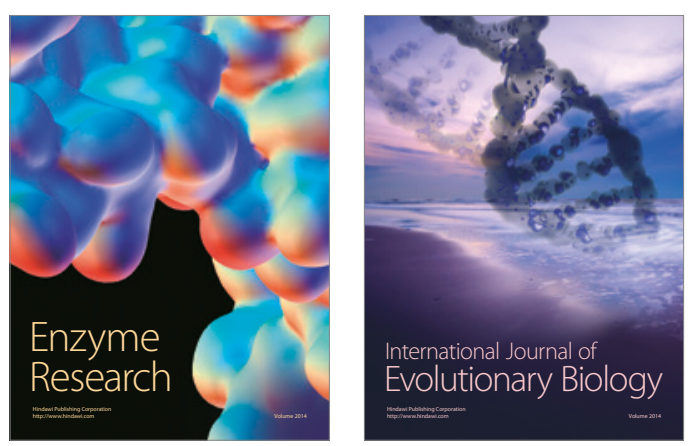
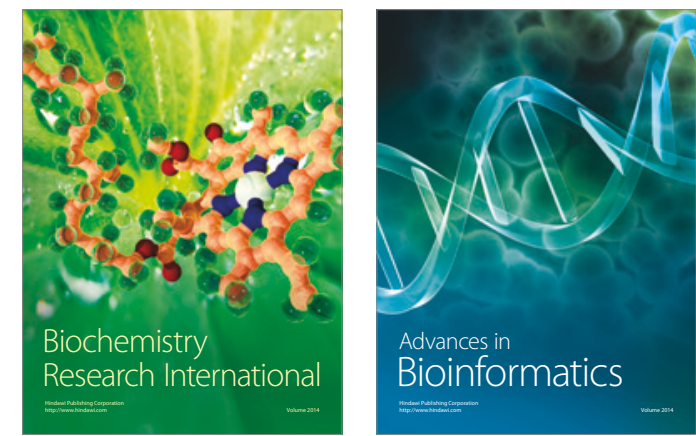

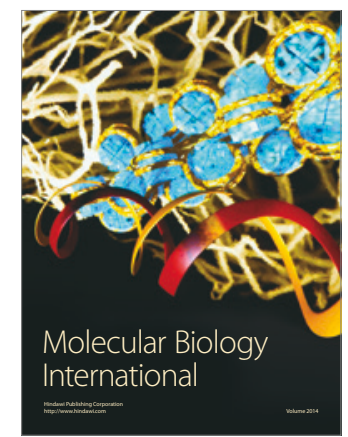

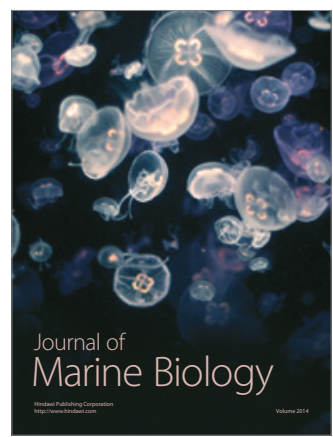

